\title{
Long non-coding RNA MIR4435-2HG promotes the progression of head and neck squamous cell carcinoma by regulating the miR-383-5p/RBM3 axis
}

\author{
SHU WANG $^{1},{\text { XIANFENG } \text { CHEN }^{2} \text { and TIANKUI QIAO }}^{1}$ \\ ${ }^{1}$ Center for Tumor Diagnosis and Therapy, Jinshan Hospital, Fudan University, Shanghai 201508; \\ ${ }^{2}$ Department of Pharmacy, Tinglin Hospital, Shanghai 201505, P.R. China \\ Received September 18, 2020; Accepted January 28, 2021
}

DOI: $10.3892 / o r .2021 .8050$

\begin{abstract}
Recent studies have shown that long non-coding RNAs (lncRNAs) are strongly related to the progression of various types of cancer. The lncRNA MIR4435-2 host gene (MIR4435-2HG) has been recently recognized as a tumor-related lncRNA that is upregulated in several tumors. However, its possible functions in head and neck squamous cell carcinoma (HNSCC) remain unclear. In tShe present study, we observed that MIR4435-2HG expression was markedly upregulated in HNSCC tissues based on a Gene Expression Profiling Interactive Analysis dataset. This result was further confirmed in HNSCC tissues and cell lines using quantitative real-time polymerase chain reaction. In addition, the high expression level of MIR4435-2HG was significantly associated with poor disease-free survival and overall survival in all HNSCC cases and was associated with advanced tumor-metastasis-node stage and poor prognosis. In vitro and in vivo assays demonstrated that MIR4435-2HG knockdown suppressed HNSCC cell proliferation and invasion, epithelial-mesenchymal transition (EMT), and tumor growth as determined by Cell Counting Kit-8, Transwell assays and western blotting. Furthermore, MIR4435-2HG affected HNSCC cell proliferation and migration and EMT by modulating the microRNA miR-383-5p to positively regulate the protein expression level of RNA-binding motif protein 3 (RBM3). In conclusion, we provide a detailed analysis of the roles of MIR4435-2HG in HNSCC and identified the MIR4435-2HG/miR-383-5p/RBM3 axis as a potential therapeutic target for HNSCC treatment.
\end{abstract}

Correspondence to: Dr Tiankui Qiao, Center for Tumor Diagnosis and Therapy, Jinshan Hospital, Fudan University, 1508 Longhang Road, Jinshan, Shanghai 201508, P.R. China

E-mail: qiaotk@163.com

Key words: MIR4435-2HG, head and neck squamous cell carcinoma, cell proliferation, cell migration, epithelial-mesenchymal transition, miR-383-5p/RBM3

\section{Introduction}

Head and neck squamous cell carcinoma (HNSCC) is the most common cancer worldwide that causes cancer-related deaths. It is an aggressive disease that is associated with high morbidity and mortality, globally affecting 600,000 new patients every year (1-3). Although there has been some progress in HNSCC therapy in recent years, the therapeutic effects of current treatment methods in patients with HNSCC remain unsatisfactory.

Long non-coding RNAs (lncRNAs) are a class of non-protein-coding transcripts that are more than 200 nucleotides in length and possess the ability to regulate gene expression (4). Accumulating evidence has shown that lncRNAs participate in multiple biological processes, including cell growth, differentiation, and metastasis (5-7). Notably, several studies have reported that IncRNAs are usually deregulated in cancers; this deregulation results in aberrant gene expression, leading to the progression of cancers, including HNSCC. For example, the IncRNA LNCAROD is upregulated in HNSCC; its knockdown represses cell proliferation and mobility in vitro and tumorigenesis in vivo (8). On the other hand, LINC00460 overexpression promotes HNSCC cell proliferation and invasion (9). The role of IncRNAs in cancer development has been the focus of several studies. However, the aberrant expression patterns and functional roles of IncRNAs in HNSCC development are still not well documented.

The lncRNA MIR4435-2 host gene (MIR4435-2HG) is located on human chromosome $2 \mathrm{q} 13$, and it is a proven oncogenic lncRNA that is implicated in various tumors (10). For example, MIR4435-2HG knockdown was shown to suppress the proliferation and invasion of ovarian cancer cells (11). MIR4435-2HG expression is upregulated in glioblastoma tissues and is associated with shorter overall survival in patients with glioblastoma (12). In addition, it serves as an oncogene in colorectal cancer, oral squamous cell carcinoma, non-small cell lung cancer, and gastric cancer $(10,13-15)$. However, the roles and mechanisms of action of MIR4435-2HG in HNSCC remain unknown.

In the present study, we found that MIR4435-2HG inhibition suppressed cell proliferation, cell migration, and epithelial-mesenchymal transition (EMT) in vitro and inhibited tumor growth in vivo. Moreover, we demonstrated that 
MIR4435-2HG serves as an oncogene in HNSCC by regulating the miR-382-5p/RNA-binding motif protein 3 (RBM3) axis. These results suggest that MIR4435-2HG functions as a potential target for cancer therapy.

\section{Materials and methods}

Tissues and cells. A total of 519 HNSCC tissues and 44 normal tissues from the Gene Expression Profiling Interactive Analysis (GEPIA)-HNSC database (http://gepia.cancer-pku.cn/) (16) were used to determine the RNA levels of MIR4435-2HG. Eighteen HNSCC specimens and paired adjacent normal tissues were acquired from patients. Our patients ranged in age from 38 to 75 , including 16 males and 2 females, and the cases were collected between June 2014 and June 2016 at Jinshan Hospital, Fudan University (Shanghai, China). This study was approved by the Institutional Review Board of Jinshan Hospital of Fudan University, and informed consent was obtained from all patients. The human HNSCC cell lines CAL27 and SCC25 were obtained from American Type Culture Collection (ATCC) and were cultured in Dulbecco's modified Eagle's medium containing 10\% fetal bovine serum (Gibco; Thermo Fisher Scientific, Inc.).

Bioinformaticsanalyses. The expression level of MIR4435-2HG in HNSCC tissues was determined using GEPIA. Correlation between the expression level of MIR4435-2HG and patients with more advanced stage or higher grade HNSCC and the prognosis based on the expression level of MIR4435-2HG were determined using GEPIA. The potential binding sites of MIR4435-2HG and miR-383-5p were predicted using miRDB (17), and those of miR-383-5p and RBM3 were predicted using TargetScan (18).

RNA extraction and quantitative real-time polymerase chain reaction $(q P C R)$. Total RNA was extracted from tissues or cells using the TRIzol reagent (Invitrogen; Thermo Fisher Scientific, Inc.) and reverse-transcribed to complementary DNA (cDNA) using the RevertAid First Strand cDNA Synthesis Kit (Thermo Fisher Scientific, Inc.). qPCR analysis was performed using Fluorescein qPCR Master Mix (Thermo Fisher Scientific, Inc.). The relative expression levels of the detected genes were determined using the $2^{-\Delta \Delta C q}$ method (19). The expression levels of MIR4435-2HG and RBM3 were normalized to those of glyceraldehyde 3-phosphate dehydrogenase, (GAPDH) and the expression level of miR-383-5p was normalized to that of U6.

Cell transfection. Short-hairpin RNA (shRNA) sequences targeting MIR4435-2HG were synthesized by Sangong Biotech (Shanghai). 293T cells were co-transfected with a validated vector and lentiviral packaging vectors $\mathrm{pMD}$ and psPAX2 using Lipofectamine 2000 (Thermo Fisher Scientific, Inc.). Virus particles were harvested and purified after $48 \mathrm{~h}$. The complete sequence of MIR4435-2HG was subcloned into a pcDNA3.1(+) vector to construct the pcDNA3.1-MIR4435-2HG vector (GenePharma). miR-383-5p mimics and their corresponding negative control (control mimics) were obtained from RiboBio. Cell transfection was performed using Lipofectamine 2000 Transfection Reagent
(Invitrogen; Thermo Fisher Scientific, Inc.) according to the manufacturer's instructions. A series of experimental operations were performed at $48 \mathrm{~h}$ after transfection.

Western blot assay. Tissues and cells were homogenized or lysed using RIPA buffer supplemented with a protease inhibitor cocktail (Roche, Applied Science). Protein concentrations was quantified by BCA protein quantification kit. Protein extracts were loaded onto $12 \%$ acrylamide gels and transferred onto polyvinylidene fluoride membranes (Millipore) for $30 \mathrm{~min}$. The membrane was blocked with a 5\% skim milk solution for $1 \mathrm{~h}$ at room temperature and incubated with the primary antibodies overnight at $4^{\circ} \mathrm{C}$. Antibodies against RBM3 (cat. no. 14363-1-AP, 1:1,000, $17 \mathrm{kDa}$ ), E-cadherin (E-cad, cat. no. 20874-1-AP, 1:10,000, $97 \mathrm{kDa}$ ), vimentin (Vim, cat. no. 60330-1-Ig, 1:5,000, $54 \mathrm{kDa}$ ), and GAPDH (cat. no. 60004-1-Ig, 1:20,000, $36 \mathrm{kDa}$ ) were acquired from Proteintech. Finally, the membrane was incubated with secondary antibodies (\#7076 and \#7074, Cell Signaling Technology) for $1 \mathrm{~h}$ at room temperature and washed three times. Protein bands were visualized using an ECL-chemiluminescence kit. Detection of intensity of the specific band was conducted by ImageJ Software (National Institutes of Health, Bethesda, MD, USA).

Cell proliferation assays. Cell viability was measured using the Cell Counting Kit-8 (CCK-8; Beyotime Institute of Biotechnology) assay. The viability of the transfected cells was determined for 5 days after plating the cells onto 96 -well plates. After incubation with the CCK-8 solution, absorbance was measured at $450 \mathrm{~nm}$. For the colony formation assay, transfected HNSCC cells were plated on to 6-well plates at a density of 300 cells per well and incubated for 12 days. The colonies were fixed, stained, and then counted under a microscope (CX21; Olympus, Tokyo, Japan) at x100 magnification.

Transwell migration and invasion assays. Cells were loaded into the upper chamber coated with Matrigel mix for the invasion assay or without the Matrigel mix for the migration assay. After incubation for $24 \mathrm{~h}$, the cells in the upper membrane of the chambers were removed using cotton wool, and the cells that had migrated or invaded through the membrane were fixed with methanol and stained with $0.5 \%$ hematoxylin. The cells were counted under a microscope (CX21; Olympus, Tokyo, Japan) in three randomly selected microscopic fields at x100 magnification.

Luciferase reporter assay. The cDNA fragments of MIR4435-2HG or 3'-untranslated region (UTR) of RBM3 containing the putative miR-383-5p binding site were inserted into pmirGLO dual luciferase vectors. CAL27 and SCC25 cells were co-transfected with wild-type (WT) or mutated (MT) MIR4435-2HG or RBM3 3'-UTR reporter plasmids and miR-383-5p mimics or control mimics. After incubation for $48 \mathrm{~h}$, the luciferase assay was performed using the Dual Luciferase Reporter Assay System (Promega Corp.).

RNA immunoprecipitation (RIP) assay. The RIP assay was performed using the Magna RIP RNA-Binding Protein Immunoprecipitation Kit (Millipore). CAL27 and SCC25 
cells were lysed with RIPA lysis buffer and then incubated with magnetic beads conjugated with antibodies against IgG (Abcam ab172730, 1:50) and AGO2 (Abcam, ab186733, 1:50). The co-precipitated RNAs were analyzed using qPCR.

HNSCC xenograft model. Four-week-old BALB/C nude mice (18-20 g, n=10) were obtained from Shanghai Laboratory Animal Center (Shanghai, China) and were conducted in accordance with the appropriate ethical standards and national guidelines, and which were used to establish the HNSCC xenograft model. CAL27 cells stably infected with shMIR4435-2HG or shNC lentivirus were subcutaneously injected into the left dorsal flanks of mice. The sizes of the xenografts were checked every week for 35 days, and the volume $(\mathrm{V})$ was determined $\left(\mathrm{V}=0.5 \mathrm{x}\right.$ length $\mathrm{x}$ width $\left.{ }^{2}\right)$ After 35 days, the mice were sacrificed by cervical dislocation. All experimental procedures and protocols were approved by the Ethics Committee of Jinshan Hospital of Fudan University.

Immunohistochemistry. Tumor tissues from the shNC and shMIR4435-2HG groups were fixed in $4 \%$ paraformaldehyde, dehydrated, embedded in paraffin, cut into $4-\mu \mathrm{m}$-thick sections, and analyzed via immunohistochemical analysis using antibodies against Ki67 (Santa Cruz Biotechnology).

Statistical analysis. Data are expressed as mean \pm standard deviation. Statistical analysis was performed using GraphPad Prism 5.0 software (GraphPad Software, Inc.). Student's t-test was used to determine significant differences between the groups. $\mathrm{P}<0.05$ was considered to indicate statistical significance.

\section{Results}

MIR4435-2HG expression is upregulated in HNSCC. To determine the expression level of MIR4435-2HG in HNSCC, we analyzed its expression in HNSCC and normal tissues using a GEPIA dataset. GEPIA analysis revealed that the expression level of MIR4435-2HG was substantially increased in HNSCC tissues compared with that in normal tissues (Fig. 1A). In addition, the higher expression level of MIR4435-2HG was associated with poorer disease-free survival and overall survival in all HNSCC cases (Fig. 1B and C). Furthermore, analysis of public data revealed that upregulated MIR4435-2HG expression was clearly discernible among the clinical stages, with considerably higher expression levels in patients with more advanced stage or higher grade HNSCC (Fig. 1D). To confirm these results, we determined the expression level of MIR4435-2HG in 18 HNSCC tissues and normal tissues using qPCR. The results showed that MIR4435-2HG expression was significantly upregulated in the tumor tissues compared with that in the normal tissues (Fig. 1E) and increased in the later stages of the cancer (Fig. 1F). We also analyzed the relationship between MIR4435-2HG level and age, sex, tumor size and lymph node metastasis stage, but there was no statistical significance (data not shown). These results suggest that MIR4435-2HG plays an important role in the tumorigenesis and progression of HNSCC.
MIR4435-2HG knockdown suppresses HNSCC cell proliferation, migration, and invasion in vitro. Next, we conducted loss-of-function assays in HNSCC cells to examine the function of MIR4435-2HG in HNSCC cells. We infected CAL27 and SCC25 cells (20-22) with lentiviral particles carrying MIR4435-2HG shRNA and successfully verified the significant decrease in the expression level of MIR4435-2HG in the established cell lines using qPCR (Fig. 2A). Then, we determined the effects of MIR4435-2HG knockdown on cell viability using the CCK-8 and colony formation assays. As shown in Fig. 2B and C, MIR4435-2HG knockdown obviously suppressed cell proliferation and resulted in a marked decrease in the colony formation ratio of CAL27 and SCC25 cells. In addition, the Transwell assay results revealed that MIR4435-2HG knockdown significantly weakened the migratory and invasive abilities of CAL27 and SCC25 cells (Fig. 2D and E). We found that E-cadherin (E-cad) expression was enhanced, whereas vimentin (Vim) expression was decreased in the CAL27 and SCC25 cells after MIR4435-2HG knockdown (Fig. 2F and G), suggesting that MIR4435-2HG knockdown inhibits EMT in HNSCC cells. Collectively, these results revealed that MIR4435-2HG negatively regulates HNSCC cell proliferation, migration, and invasion in vitro.

MIR4435-2HG knockdown attenuates tumor growth in vivo. To further investigate the tumorigenic effects of MIR4435-2HG on HNSCC cells in vivo, we established a tumor xenograft mouse model by subcutaneously inoculating CAL27 cells into $\mathrm{BALB} / \mathrm{C}$ nude mice according to the in vitro results and based on reports as previously described (23-25). As shown in Fig. 3A-C, both the volumes and weights of the tumors in the shMIR4435-2HG group were significantly decreased compared with those in the shNC group. Immunohistochemical staining for the cell proliferation marker Ki67 revealed lower percentages of Ki67-positive tumor cells and showed the membrane localization of E-cad as well as lower expression of Vim in the shMIR4435-2HG group compared with that in the shNC group (Fig. 3D). Western blot analysis revealed that E-cad expression was enhanced, whereas Vim expression levels were reduced in the shMIR4435-2HG group (Fig. 3E). These results indicate that MIR4435-2HG enhances the tumorigenicity of HNSCC cells in vivo.

MIR4435-2HG acts as a competing endogenous RNA (ceRNA) and competitively binds miR-383-5p. Using the online software miRDB, we found that MIR4435-2HG forms complementary base pairs with miR-383-5p (Fig. 4A). We first performed the luciferase reporter assay to confirm the direct binding between MIR4435-2HG and miR-383-5p. The results showed that HNSCC cells transfected with miR-383-5p mimics had significantly decreased luciferase activity compared with those transfected with MIR4435-2HG-WT; on the other hand, the luciferase activities of HNSCC cells transfected with miR-383-5p mimics and MIR4435-2HG-MT remained unaffected (Fig. 4B). Furthermore, we examined miR-383-5p and MIR4435-2HG on magnetic beads conjugated to the anti-Ago2 antibody. RIP assay results showed that MIR4435-2HG was preferentially enriched in Ago2-containing miRNPs compared with than in control 
A

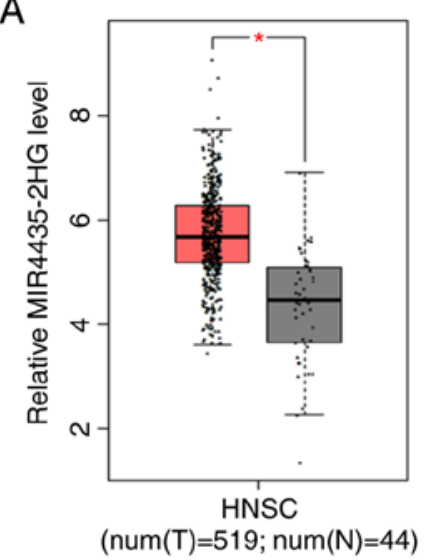

B

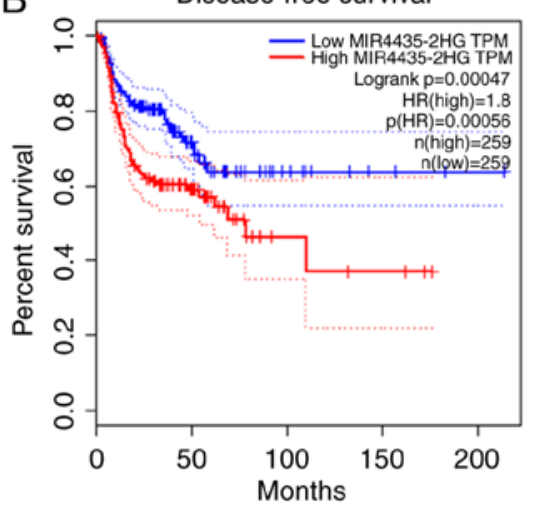

C

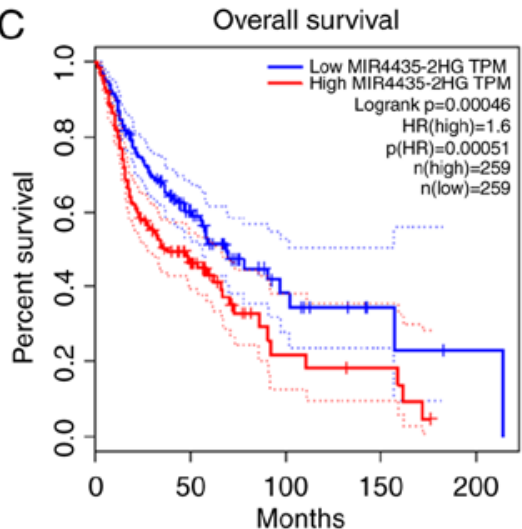

$\mathrm{F}$

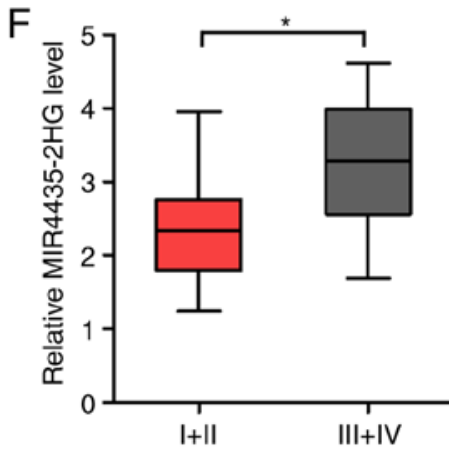

Figure 1. MIR4435-2HG expression is upregulated in HNSCC tissues. (A) The expression level of MIR4435-2HG in 519 HNSCC tissues and 44 normal tissues was analyzed using a GEPIA database. (B and C) Prognostic significance of MIR4435-2HG in HNSCC tissues obtained from the GEPIA database. (D) The expression level of MIR4435-2HG in different clinical stages of HNSCC from the GEPIA database. (E) The expression level of MIR4435-2HG in 18 HNSCC tissue samples and adjacent normal tissues was determined using qPCR. (F) The expression level of MIR4435-2HG in HNSCC tissues from patients with different clinical stages was determined. ${ }^{*} \mathrm{P}<0.05$ and ${ }^{* *} \mathrm{P}<0.01$. MIR4435-2HG, lncRNA MIR4435-2 host gene; HNSCC, head and neck squamous cell carcinoma.

IgG immunoprecipitates. Similarly, miR-383-5p was detected at a higher level than control anti-IgG (Fig. 4C). Therefore, MIR4435-2HG is present in Ago2-containing miRNPs, possibly by associating with miR-383-5p. This result is consistent with our bioinformatics analysis and luciferase assay results. In addition, MIR4435-2HG knockdown significantly increased miR-383-5p expression (Fig. 4D). These results suggest that MIR4435-2HG acts as a sponge for miR-383-5p in HNSCC cells.

$R B M 3$ is a target of $m i R-383-5 p$. We identified RBM3 as a potential target of miR-383-5p using TargetScan (Fig. 5A). After co-transfection with luciferase reporter vectors and miR-383-5p mimics, the luciferase activity of RBM3-WT was significantly reduced, whereas the activity of RBM3-MT was unaffected by the miR-383-5p mimic (Fig. 5B). Subsequent experiments revealed that miR-383-5p overexpression significantly reduced RBM3 expression at the mRNA and protein levels (Fig. 5C-E) in the CAL27 and SCC25 cell lines. These data suggest that miR-383-5p inhibits RBM3 expression in HNSCC cells by directly targeting the 3'-UTR of RBM3.

miR-383-5p reverses the tumor-promoting roles of MIR4435-2HG in HNSCC cells by regulating RBM3. Recent studies have shown that lncRNAs function as ceRNAs by competitively binding with miRNAs during tumorigenesis. We speculated that MIR4435-2HG affects the biological behavior of HNSCC cells by regulating the miR-383-5p/RBM3 axis .
Western blotting showed that MIR4435-2HG overexpression promoted RBM3 expression in HNSCC cells; however, co-transfection with miR-383-5p mimics reduced this effect (Fig. 6A). This result indicates that MIR4435-2HG regulates the protein expression level of RBM3 by influencing miR-383-5p expression in HNSCC cells. In addition, we observed that the transfection of HNSCC cells with miR-383-5p mimics abrogated the promotive effects of MIR4435-2HG on cell colony formation, migration, and invasion (Fig. 6B-E). Moreover, we observed that MIR4435-2HG decreased E-cad expression and increased Vim expression; these changes were abolished in HNSCC cells co-transfected with MIR4435-2HG and miR-383-5p (Fig. 6F). These results suggest that miR-383-5p reverses the function of MIR4435-2HG in HNSCC development by regulating RBM3 expression.

\section{Discussion}

Recently, studies have shown that the lncRNA MIR4435-2 host gene (MIR4435-2HG) is abnormally expressed and functions as an oncogene in ovarian cancer (11), colorectal cancer (13), gastric cancer (10), and hepatocellular carcinoma (26); however, its function in head and neck squamous cell carcinoma (HNSCC) remains unknown. In the present study, we showed that the expression level of MIR4435-2HG was upregulated in HNSCC tissues and cell lines. MIR4435-2HG knockdown suppressed HNSCC cell proliferation, migration and invasion. 

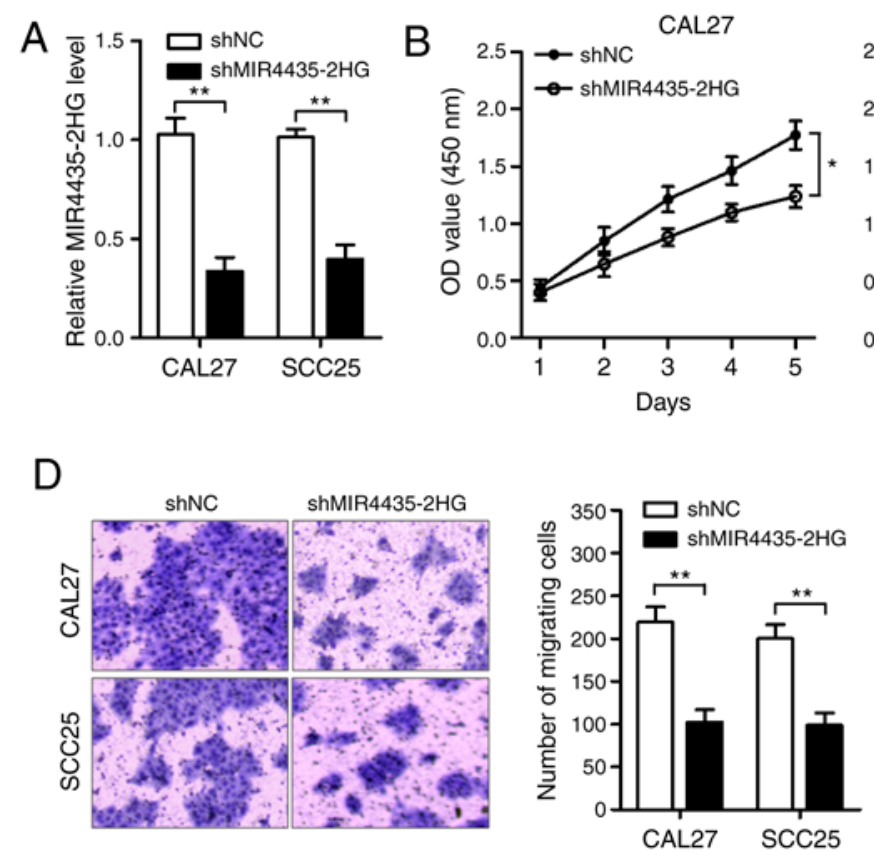

E
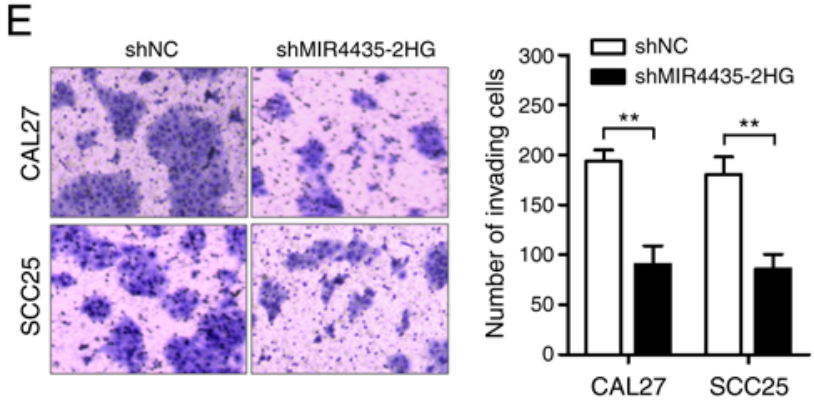

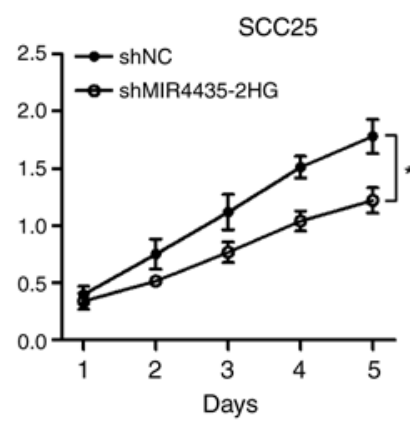

F

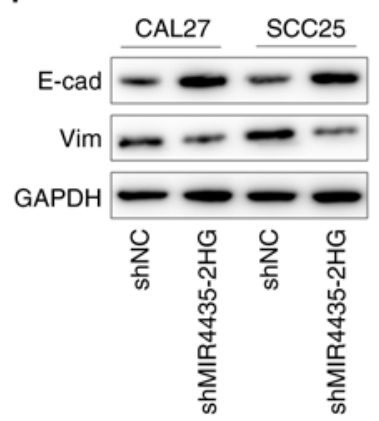

G

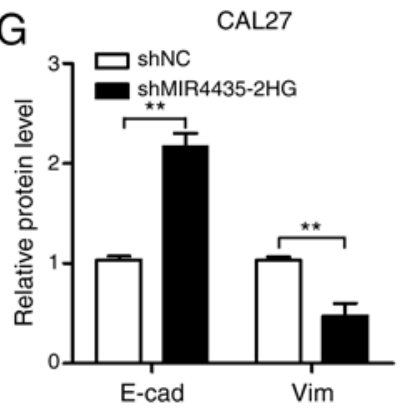

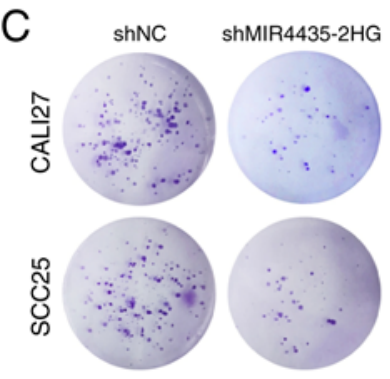
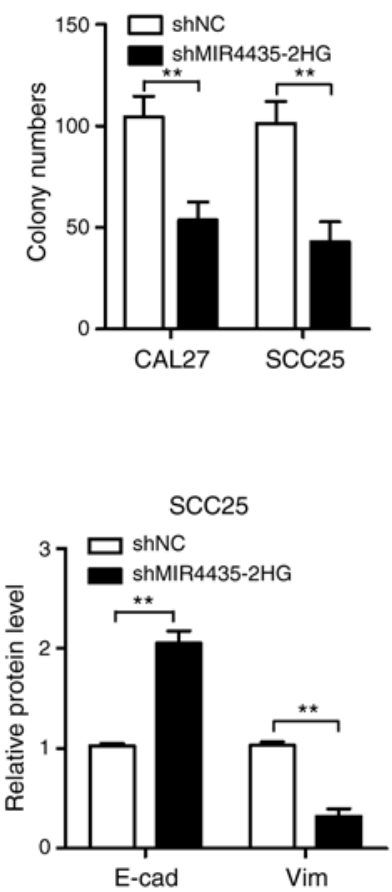

Figure 2. MIR4435-2HG knockdown suppresses HNSCC cell proliferation, migration, and invasion in vitro. (A) CAL27 and SCC25 cells were infected with MIR4435-2HG shRNA (shMIR4435-2HG) or control shRNA (shNC), respectively, and qPCR was used to determine the expression level of MIR4435-2HG. (B) Cell viability was determined using the CKK-8 assay. (C) Cell proliferation was determined using the colony formation assay. (D) Cell migration was determined using the Transwell migration assay. (E) Cell invasion was determined using the Transwell invasion assay. (F and G) The protein expression levels of E-cadherin (E-cad) and vimentin (Vim) were determined using western blot analysis. ${ }^{*} \mathrm{P}<0.05$ and ${ }^{* *} \mathrm{P}<0.01$. MIR4435-2HG, lncRNA MIR4435-2 host gene; HNSCC, head and neck squamous cell carcinoma.

Epithelial-mesenchymal transition (EMT) is a process in which cancer cells lose their polarity and cell-cell adhesion, develop into mesenchymal fibroblast-like cells, and then confer metastatic properties to cancer cells by increasing their ability to migrate and invade. In cancer cells, EMT is a complex reprogramming process characterized by inhibition of the above cortical markers and upregulation of mesenchymal markers (27). Thus, we also determined the role of MIR4435-2HG on HNSCC cell EMT and found that MIR4435-2HG knockdown inhibited HNSCC cell EMT by enhancing E-cadherin and reducing vimentin expression levels. Based on the results of previous research (13), MIR4435-2HG may serve as an miRNA sponge and exhibit its functions in several tumor types. We further revealed that the MIR4435-2HG/miR-383-5p/RBM3 axis is a potential competing endogenous RNA (ceRNA) regulatory network in HNSCC.

Increasing evidence shows that lncRNAs can serve as ceRNAs in various cancers. For example, the lncRNA LINC00460 promotes tumor growth and metastasis in patients with hepatocellular carcinoma by upregulating the expression of miR-342-3p-dependent AGR2 (28). The lncRNA OGFRP1 acts as a ceRNA to facilitate prostate cancer progression by regulating the expression level of SARM1 via miR-124-3p (29). The lncRNA HNF1A-AS1 serves as a ceRNA in gastric cancer by promoting the activation of the PI3K/AKT signaling pathway by sponging miR-30b-3p (30). MIR4435-2HG has also been observed to play a role in several cancers through this regulatory mechanism. For example, MIR4435-2HG knockdown suppressed ovarian cancer cell proliferation, invasion, and migration through the miR-128-3p/CDK14 axis (11), MIR4435-2HG promoted the proliferation and invasion of glioblastoma cells by regulating the miR-1224-5p/TGFBR2 axis (12), and MIR4435-2HG acted as a ceRNA to upregulate YAP1 expression by sponging miR-206 and promoted colorectal cancer growth and metastasis (13). To investigate the mechanism of MIR4435-2HG regulation in HNSCC, bioinformatics analysis indicated that miR-383-5p, a tumor suppressor in several types of cancers, including triple-negative breast cancer, ovarian cancer, and gastric cancer (31-33), has potential MIR4435-2HG binding sites. In the present study, 

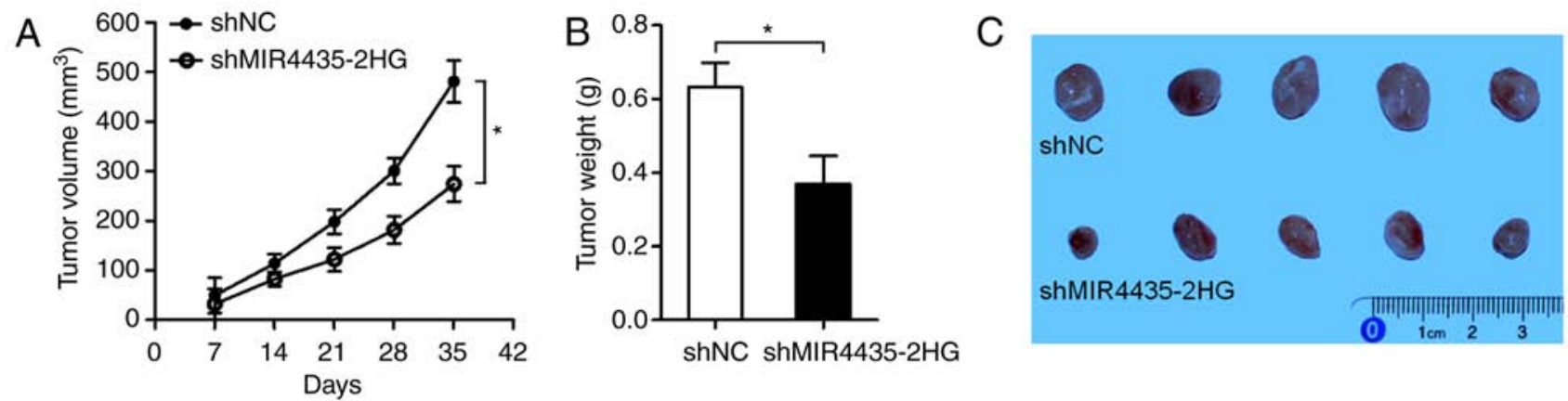

D
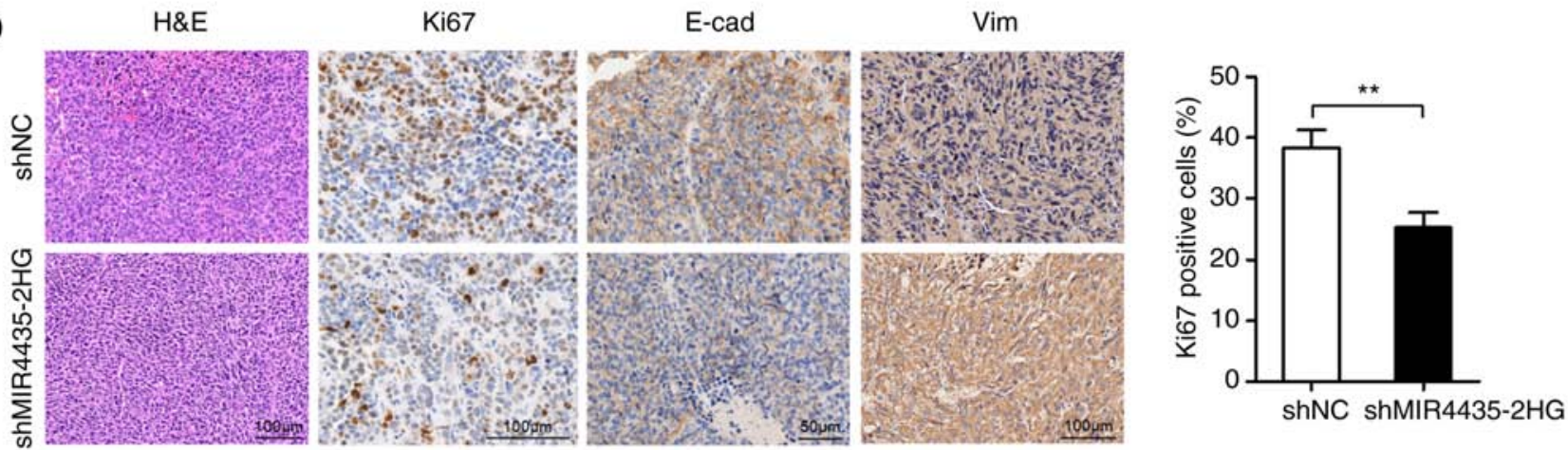

E
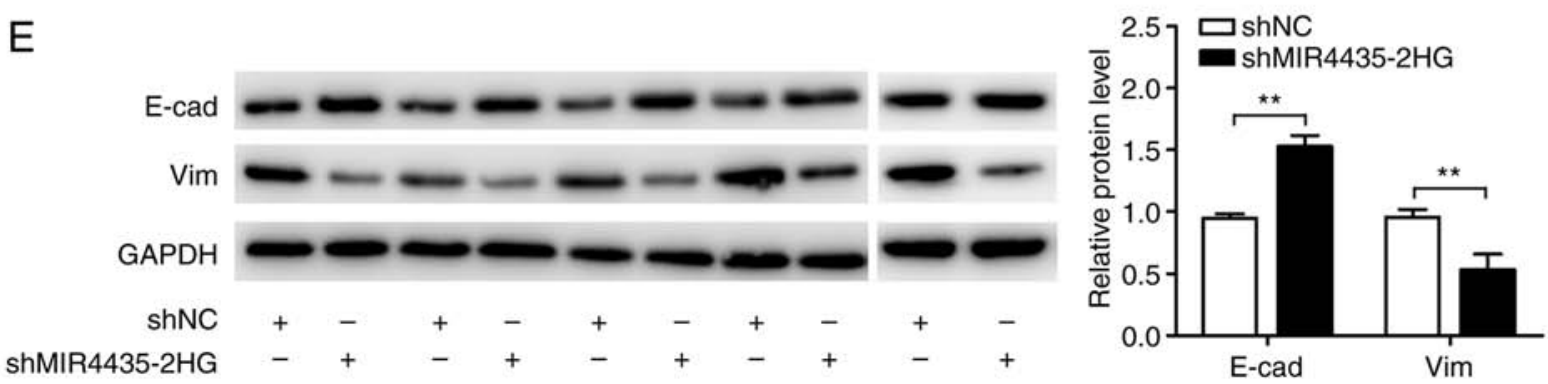

Figure 3. MIR4435-2HG knockdown inhibits HNSCC tumor growth in vivo. The in vivo xenograft assay was performed using MIR4435-2HG-knockdown CAL27 cells or control cells. (A and B) Tumor growth (volume in A and weight in B) was determined in the indicated groups. (C) Representative images of the xenograft tumors. (D) Representative image of hematoxylin and eosin (H\&E) staining and immunohistochemical staining with Ki67, E-cadherin (E-cad) and vimentin (Vim) antibodies. (E) The protein expression levels of E-cad and Vim were determined using western blot analysis. ${ }^{*} \mathrm{P}<0.05$ and ${ }^{* *} \mathrm{P}<0.01$ MIR4435-2HG, lncRNA MIR4435-2 host gene; HNSCC, head and neck squamous cell carcinoma.

we demonstrated that MIR4435-2HG serves as a ceRNA and competitively binds miR-383-5p using luciferase reporter and RIP assays. In addition, we demonstrated that MIR4435-2HG knockdown induces miR-383-5p expression. These results confirm that miR-383-5p is a direct target of MIR4435-2HG.

RNA-binding motif protein 3 (RBM3) is an RNA- and DNA-binding protein whose gene encodes two alternatively spliced RNA transcripts and maps to Xp11.23 (34). RBM3 plays a key role in tumor progression. In a previous study, RBM3 was shown to promote the proliferation of hepatocellular carcinoma cells in an SCD-circRNA 2-dependent manner (35). RBM3 expression was found to be upregulated in human breast cancer tissues compared with that in adjacent normal tissues (36). RBM3 also exhibited this function in other cancers such as glioblastoma, non-small cell lung cancer, and urinary bladder cancer (37-39). In addition, it has been reported to act as a target of miR-383-5p in triple-negative breast cancer (31). In the present study, bioinformatics analysis and luciferase reporter assay results showed that RBM3 could function as a direct target of miR-383-5p in HNSCC. Moreover, we demonstrated that miR-383-5p mimics decreased RBM3 expression in HNSCC cells, which was attenuated by MIR4435-2HG. Furthermore, miR-383-5p mimics abrogated the promotive effects of MIR4435-2HG on cell proliferation, migration, invasion, and EMT. These results suggest that RBM3 is involved in the effect of MIR4435-2HG in HNSCC, and the detailed function of RBM3 in HNSCC will be explored in subsequent research.

We demonstrated that MIR4435-2HG affected HNSCC progression partly through regulating the miR-383-5p/RBM3 axis. Through bioinformatics analysis, it can be predicted that MIR4435-2HG can bind to multiple miRNAs, and one miRNA can also bind to multiple target mRNAs. In this study, we only selected a potential miRNA and mRNA that play a role in HNSCC, confirming that MIR4435-2HG can partially affect the HNSCC invasion process by regulating the miR-383-5p/RBM3 axis. In our next study, we will continue to study the role of MIR4435-2HG in HNSCC, further identify potential miRNAs and mRNAs by MIR4435-2HG, and their roles in HNSCC. In subsequent research, we also will focus 
A

miR-383-5p
${ }^{-} \mathrm{c}$ a

cuc uc gaucag ggug uuguggcu ggu u

||| || |||||| |||| ||||||| |||

gag ag cugguc ucac gacaccga cua a

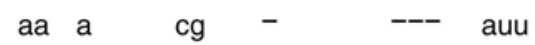

MIR4435-2HG
$\mathrm{B}$

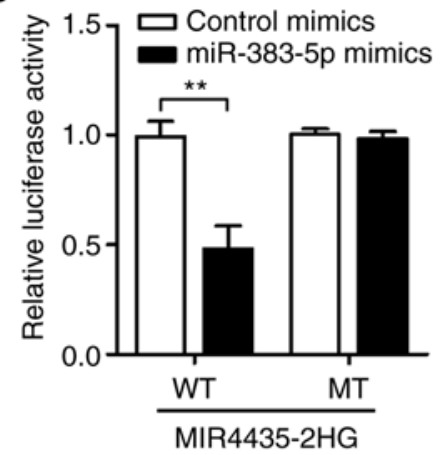

$\operatorname{scC} 25$

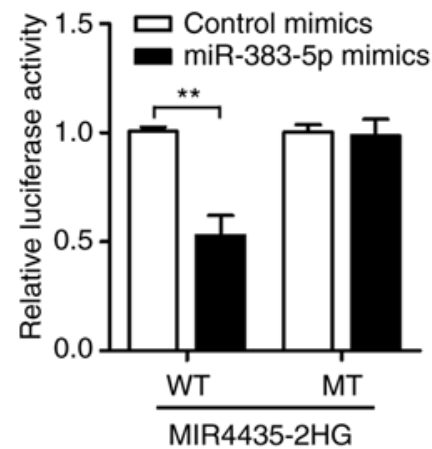

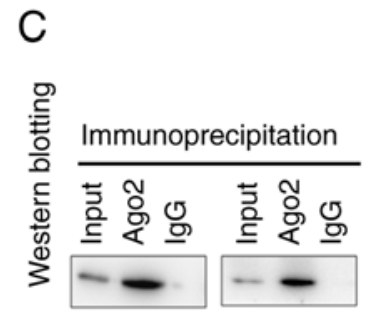
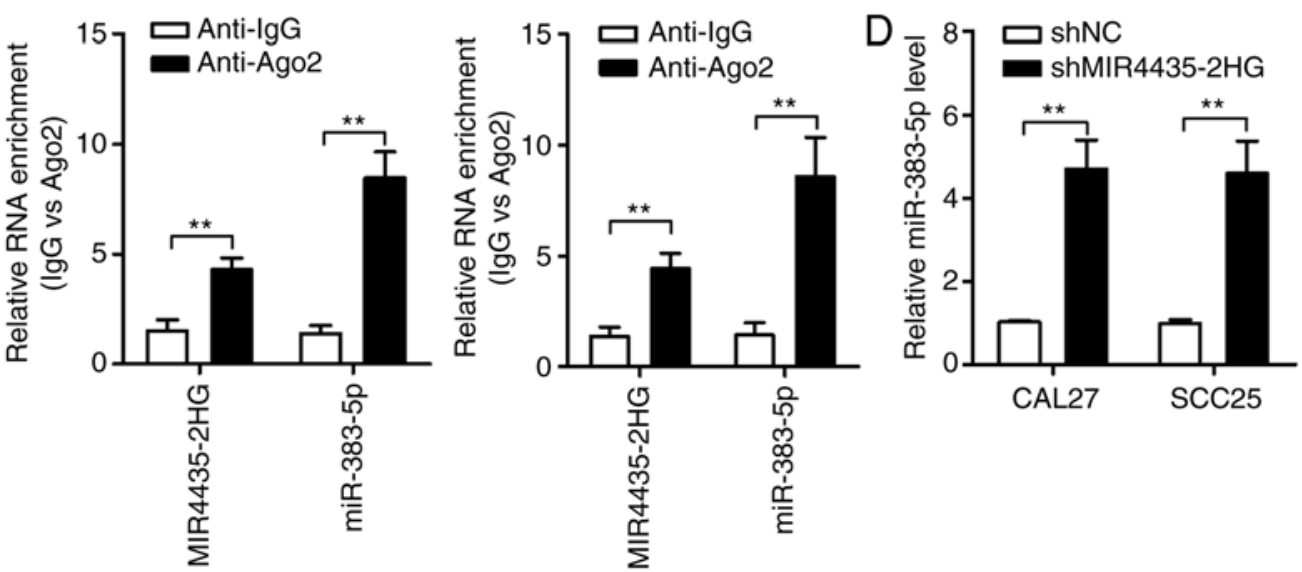

Figure 4. MIR4435-2HG serves as a miR-383-5p sponge and inhibits its expression in HNSCC cells. (A) The binding site of miR-383-5p was predicted using the miRDB program. (B) The luciferase activity of HNSCC cells transfected with miR-383-5p mimic and MIR4435-2HG-WT (or MIR4435-2HG-MT) was determined. (C) RNA immunoprecipitation (RIP) assay was performed using cell lysates, normal IgG, or anti-Ago2 antibodies. The relative expression levels of MIR4435-2HG and miR-383-5p were determined using qPCR. (D) The expression level of miR-383-5p in shMIR4435-2HG (or shNC)-transfected CAL27 and SCC25 cells was determined using qPCR. ${ }^{* *} \mathrm{P}<0.01$. MIR4435-2HG, 1ncRNA MIR4435-2 host gene; HNSCC, head and neck squamous cell carcinoma.

A

Predicted pairing of target region (top) and miRNA (bottom)

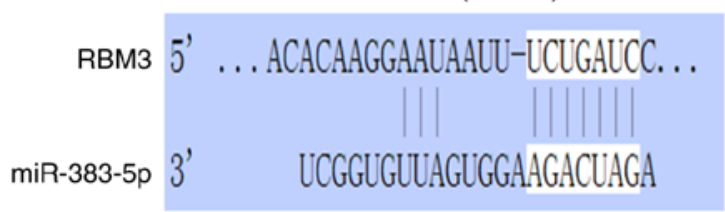

CAL27

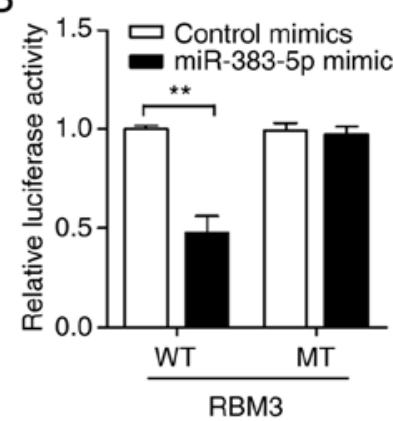

$\operatorname{SCC} 25$

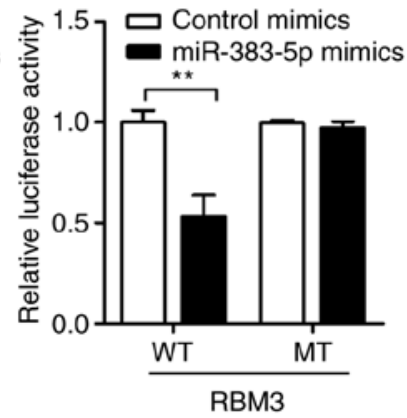

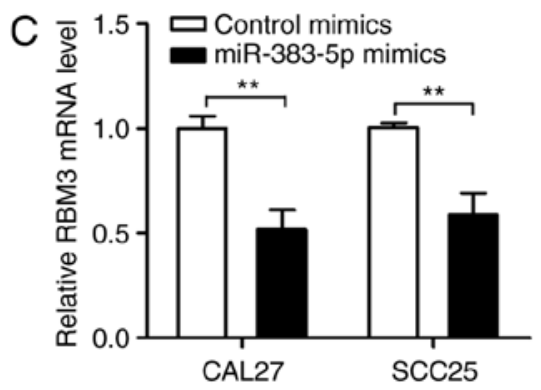
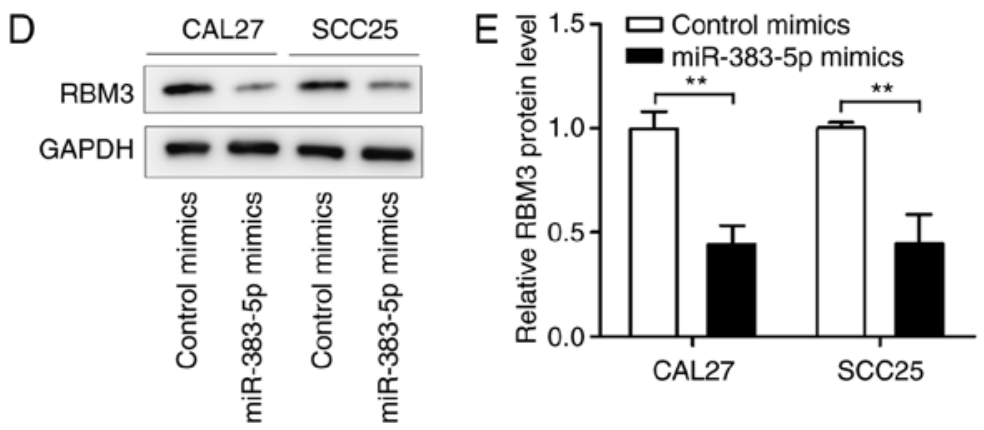

Figure 5. RBM3 is a target of miR-383-5p. (A) The 3'-UTR (untranslated region) of RBM3 is potentially targeted by miR-383-5p, as predicted using TargetScan. (B) The relative luciferase activity of HNSCC cells after co-transfection with wild-type (WT) or mutant (MT) RBM3 3'-UTR reporter genes and miR-383-5p mimics or control mimics. (C) The mRNA expression level of RBM3 in HNSCC cells transfected with miR-383-5p mimics or control mimics was analyzed using qPCR. (D and E) The protein expression level of RBM3 in HNSCC cells transfected with miR-383-5p mimics or control mimics was measured using western blot analysis. ${ }^{* *} \mathrm{P}<0.01$. RBM3, RNA-binding motif protein 3 ; HNSCC, head and neck squamous cell carcinoma. 
A

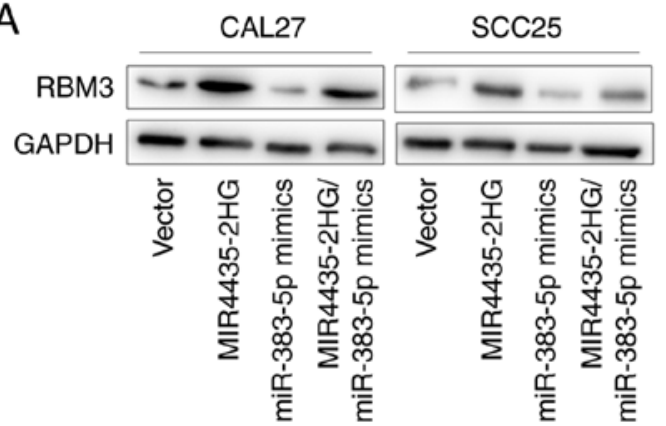

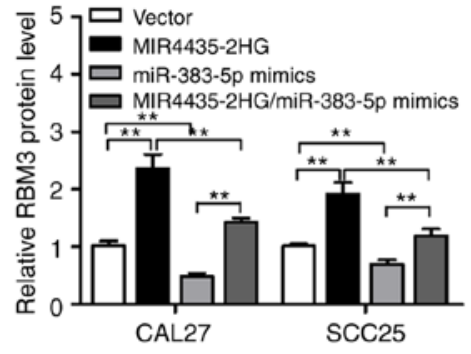

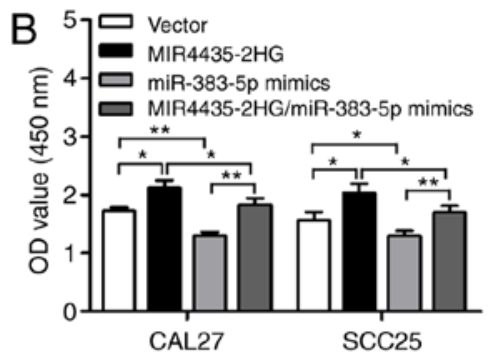

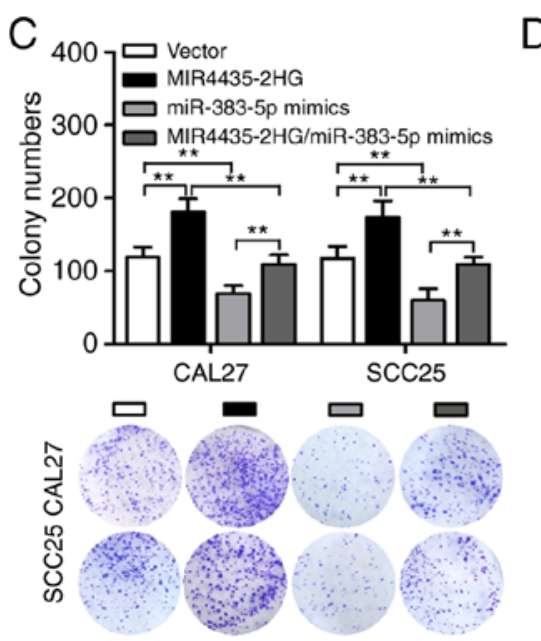

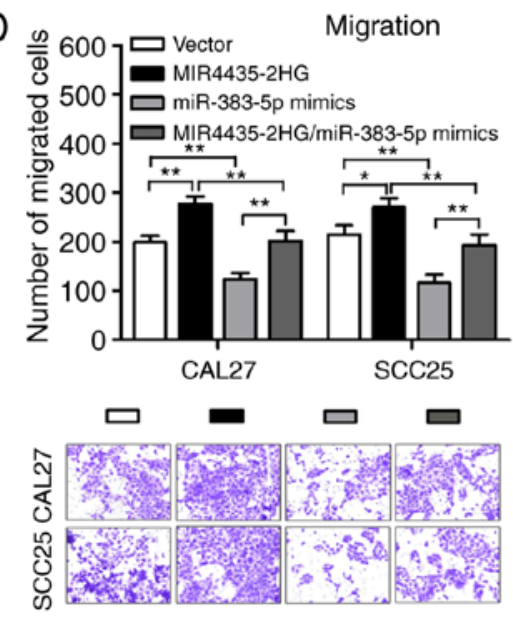

E

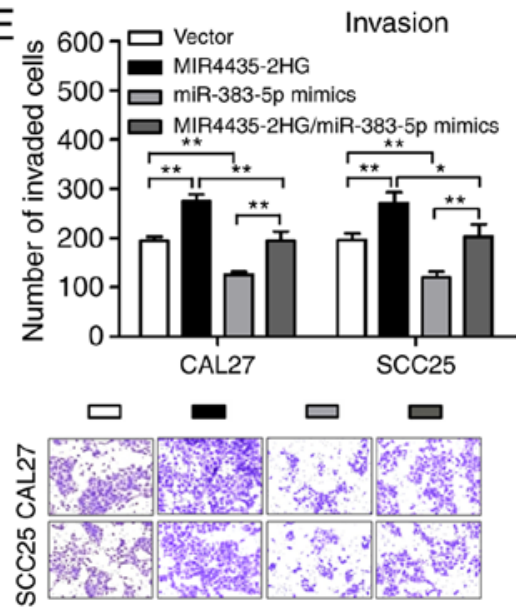

$\mathrm{F}$

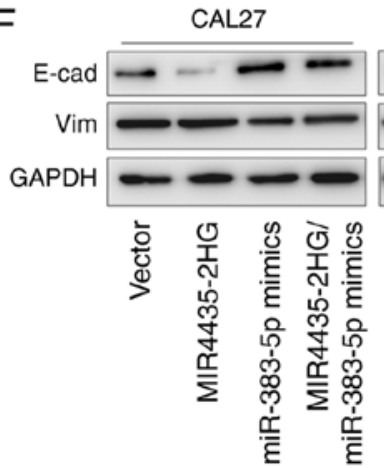

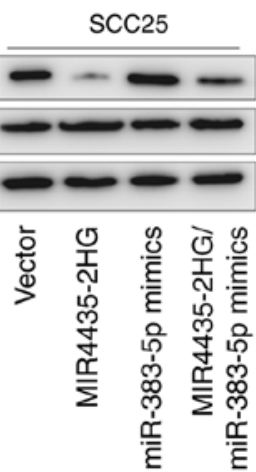
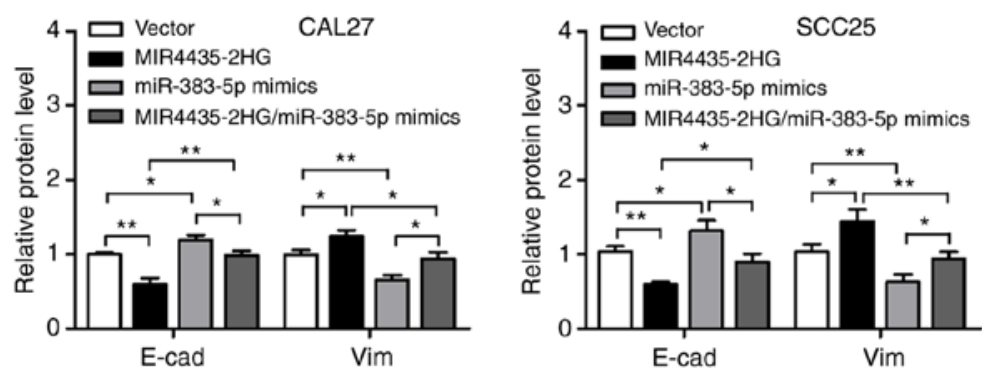

Figure 6. miR-383-5p abolishes the function of MIR4435-2HG in HNSCC cells by regulating RBM3 expression. (A) The protein expression level of RBM3 was determined in HNSCC cells transfected with MIR4435-2HG, miR-383-5p mimics alone, or co-transfected with MIR4435-2HG and miR-383-5p mimics. (B) Cell proliferation, (C) cell colony formation, (D) cell migration, and (E) cell invasion were detected in the different groups. (F) The protein expression levels of E-cad (E-cadherin) and Vim (vimentin) were measured using western blotting. ${ }^{*} \mathrm{P}<0.05$ and ${ }^{* *} \mathrm{P}<0.01$. MIR4435-2HG, lncRNA MIR4435-2 host gene; RBM3, RNA-binding motif protein 3; HNSCC, head and neck squamous cell carcinoma.

on the impact of MIR4435-2HG on metastasis and the expression pattern of EMT marker genes should be examined in the presence of TGF- $\beta$, as TGF- $\beta 1$ could induce EMT of HNSCC cells. In conclusion, we found that MIR4435-2HG expression is upregulated in HNSCC cells and that its knockdown suppresses cell proliferation, invasion, and EMT in vitro and inhibits tumor growth and EMT in vivo. Mechanistically, the MIR4435-2HG/miR-383-5p/RBM3 axis may play key roles in HNSCC progression, thereby functioning as a novel therapeutic target for cancer treatment.

\section{Acknowledgements}

Not applicable.

\section{Funding}

This research was supported by the Jinshan Health Planning Committee Fund (grant no. JSKJ-KTMS-2018-06) and Youth Project Initiation Fund from Jinshan Hospital, Fudan University (grant no. JYQN-JC-202103). 


\section{Availability of data and materials}

The datasets used during the present study are available from the corresponding author upon reasonable request.

\section{Authors' contributions}

SW and TQ contributed to the study conception and design. SW and XC performed the experiments, analyzed the data and wrote the manuscript. TQ revised this manuscript. All authors read and approved the final manuscript.

\section{Ethics approval and consent to participate}

This study was approved by the Institutional Review Board of Jinshan Hospital of Fudan University, and informed consent was obtained from all patients. Moreover, all experimental procedures and protocols for the animal study were approved by the Ethics Committee of Jinshan Hospital of Fudan University (JIEC 2021-S20).

\section{Patient consent for publication}

Not applicable.

\section{Competing interests}

The authors declare that they have no competing interests.

\section{References}

1. Rothenberg SM and Ellisen LW: The molecular pathogenesis of head and neck squamous cell carcinoma. J Clin Invest 122: 1951-1957, 2012.

2. Tuttle TR, Mierzwa ML, Wells SI, Fox SR and Ben-Jonathan N: The cyclic GMP/protein kinase G pathway as a therapeutic target in head and neck squamous cell carcinoma. Cancer Lett 370 : 279-285, 2016.

3. Leemans CR, Braakhuis BJ and Brakenhoff RH: The molecular biology of head and neck cancer. Nat Rev Cancer 11: 9-22, 2011.

4. Lan T, Ma W, Hong Z, Wu L, Chen X and Yuan Y: Long non-coding RNA small nucleolar RNA host gene 12 (SNHG12) promotes tumorigenesis and metastasis by targeting miR-199a/ b-5p in hepatocellular carcinoma. J Exp Clin Cancer Res 36: 11, 2017.

5. Liu S, Zhang J, Yin L, Wang X, Zheng Y, Zhang Y, Gu J, Yang L, Yang J, Zheng P, et al: The lncRNA RUNX1-IT1 regulates C-FOS transcription by interacting with RUNX1 in the process of pancreatic cancer proliferation, migration and invasion. Cell Death Dis 11: 412, 2020.

6. Zhong J, Tu X, Kong Y, Guo L, Li B, Zhong W, Cheng Y, Jiang Y and Jiang Q: LncRNA H19 promotes odontoblastic differentiation of human dental pulp stem cells by regulating miR-140-5p and BMP-2/FGF9. Stem Cell Res Ther 11: 202, 2020.

7. Wang Q, Wu J, Huang H, Jiang Y, Huang Y, Fang H, Zheng G, Zhou X, Wu Y, Lei C and Hu D: IncRNA LIFR-AS1 suppresses invasion and metastasis of non-small cell lung cancer via the miR-942-5p/ZNF471 axis. Cancer Cell Int 20: 180, 2020.

8. Ban Y, Tan P, Cai J,Li J, Hu M, Zhou Y, Mei Y, Tan Y, $\mathrm{Li} X$, Zeng Z, et al: LNCAROD is stabilized by m6A methylation and promotes cancer progression via forming a ternary complex with HSPA1A and YBX1 in head and neck squamous cell carcinoma. Mol Oncol 14: 1282-1296, 2020.

9. Xie X, Xiong G, Wang Q, Ge Y and Cui X: Long non-coding RNA LINC00460 promotes head and neck squamous cell carcinoma cell progression by sponging miR-612 to up-regulate AKT2. Am J Transl Res 11: 6326-6340, 2019.
10. Wang H, Wu M, Lu Y,He K, Cai X, Yu X, Lu J and Teng L: LncRNA MIR4435-2HG targets desmoplakin and promotes growth and metastasis of gastric cancer by activating $\mathrm{Wnt} / \beta$-catenin signaling. Aging (Albany NY) 11: 6657-6673, 2019.

11. Zhu L, Wang A, Gao M, Duan X and Li Z: LncRNA MIR4435-2HG triggers ovarian cancer progression by regulating miR-128-3p/CKD14 axis. Cancer Cell Int 20: 145, 2020.

12. Xu H, Zhang B, Yang Y, Li Z, Zhao P, Wu W, Zhang $\mathrm{H}$ and Mao J: LncRNA MIR4435-2HG potentiates the proliferation and invasion of glioblastoma cells via modulating miR-1224-5p/TGFBR2 axis. J Cell Mol Med 24: 6362-6372, 2020.

13. Dong X, Yang Z, Yang H, Li D and Qiu X: Long non-coding RNA MIR4435-2HG promotes colorectal cancer proliferation and metastasis through miR-206/YAP1 Axis. Front Oncol 10: $160,2020$.

14. Zhang S, Li C, Liu J, Geng F, Shi X, Li Q, Lu Z and Pan Y: Fusobacterium nucleatum promotes epithelial-mesenchymal transiton through regulation of the lncRNA MIR4435-2HG/ miR-296-5p/Akt2/SNAI1 signaling pathway. FEBS J 287: 4032-4047, 2020.

15. Yang $M$, He X, Huang X, Wang J, He Y and Wei L: LncRNA MIR4435-2HG-mediated upregulation of TGF- $\beta 1$ promotes migration and proliferation of nonsmall cell lung cancer cells. Environ Toxicol 35: 582-590, 2020.

16. Tang Z, Li C, Kang B, Gao G, Li C and Zhang Z: GEPIA: A web server for cancer and normal gene expression profiling and interactive analyses. Nucleic Acids Res 45: W98-W102, 2017.

17. Chen Y and Wang X: miRDB: An online database for prediction of functional microRNA targets. Nucleic Acids Res 48: D127-D131, 2020.

18. Agarwal V, Bell GW, Nam JW and Bartel DP: Predicting effective microRNA target sites in mammalian mRNAs. Elife 4: e05005, 2015.

19. Livak KJ and Schmittgen TD: Analysis of relative gene expression data using real-time quantitative PCR and the 2(-Delta Delta C(T)) method. Methods 25: 402-408, 2001.

20. Foki E, Stanisz I, Kadletz L, Kotowski U, Seemann R, Schmid R and Heiduschka G: HS-173, a selective PI3K inhibitor, induces cell death in head and neck squamous cell carcinoma cell lines. Wien Klin Wochenschr 133: 26-31, 2021.

21. Khalil A and Jameson MJ: Downregulation of IGF1R expression inhibits growth and enhances cisplatin sensitivity of head and neck squamous cell carcinoma cells in vitro. Horm Cancer 10: $11-23,2019$

22. Li SJ, Yang XN and Qian HY: Antitumor effects of WNT2B silencing in GLUT1 overexpressing cisplatin resistant head and neck squamous cell carcinoma. Am J Cancer Res 5: 300-308, 2014.

23. Zhuang Z, Yu P, Xie N, Wu Y, Liu H, Zhang M, Tao Y, Wang W, Yin H, Zou B, et al: MicroRNA-204-5p is a tumor suppressor and potential therapeutic target in head and neck squamous cell carcinoma. Theranostics 10: 1433-1453, 2020.

24. Jiang Y, Cao W, Wu K, Qin X, Wang X, Li Y, Yu B, Zhang Z, Wang X, Yan M, et al: LncRNA LINC00460 promotes EMT in head and neck squamous cell carcinoma by facilitating peroxiredoxin-1 into the nucleus. J Exp Clin Cancer Res 38: 365, 2019.

25. Wu Y, Wang Y, Diao P, Zhang W, Li J, Ge H, Song Y, Li Z, Wang D, Liu L, et al: Therapeutic targeting of BRD4 in head neck squamous cell carcinoma. Theranostics 9: 1777-1793, 2019.

26. Kong Q, Liang C, Jin Y, Pan Y, Tong D, Kong Q and Zhou J: The lncRNA MIR4435-2HG is upregulated in hepatocellular carcinoma and promotes cancer cell proliferation by upregulating miRNA-487a. Cell Mol Biol Lett 24: 26, 2019.

27. Galle E, Thienpont B, Cappuyns S, Venken T, Busschaert P, Van Haele M, Van Cutsem E, Roskams T, van Pelt J, Verslype C, et al: DNA methylation-driven EMT is a common mechanism of resistance to various therapeutic agents in cancer. Clin Epigenetics 12: 27, 2020.

28. Hong H, Sui C, Qian T, Xu X, Zhu X, Fei Q, Yang J and $\mathrm{Xu}$ M: Long noncoding RNA LINC00460 conduces to tumor growth and metastasis of hepatocellular carcinoma through miR-342-3p-dependent AGR2 up-regulation. Aging (Albany NY) 12: 10544-10555, 2020.

29. Yan K, Hou L, Liu T, Jiao W, Ma Q, Fang Z, Zhang S, Song D, Liu J, Gao X and Fan Y: lncRNA OGFRP1 functions as a ceRNA to promote the progression of prostate cancer by regulating SARM1 level via miR-124-3p. Aging (Albany NY) 12: 8880-8892, 2020. 
30. Liu HT, Ma RR, Lv BB, Zhang H, Shi DB, Guo XY, Zhang GH and Gao P: LncRNA-HNF1A-AS1 functions as a competing endogenous RNA to activate PI3K/AKT signalling pathway by sponging miR-30b-3p in gastric cancer. Br J Cancer 122: 1825-1836, 2020.

31. Tian Y, Xia S, Ma M and Zuo Y: LINC00096 promotes the proliferation and invasion by sponging miR-383-5p and regulating $\mathrm{RBM} 3$ expression in triple-negative breast cancer. Onco Targets Ther 12: 10569-10578, 2019.

32. Jiang J, Xie C, Liu Y, Shi Q and Chen Y: Up-regulation of miR-383-5p suppresses proliferation and enhances chemosensitivity in ovarian cancer cells by targeting TRIM27. Biomed Pharmacother 109: 595-601, 2019.

33. Wei C and Gao JJ: Downregulated miR-383-5p contributes to the proliferation and migration of gastric cancer cells and is associated with poor prognosis. PeerJ 7: e7882, 2019.

34. Gao G, Shi X, Long Y, Yao Z, Shen J and Shen L: The prognostic and clinicopathological significance of RBM3 in the survival of patients with tumor: A Prisma-compliant meta-analysis. Medicine 99: e20002, 2020.

35. Dong W, Dai ZH, Liu FC, Guo XG, Ge CM, Ding J, Liu H and Yang F: The RNA-binding protein RBM3 promotes cell proliferation in hepatocellular carcinoma by regulating circular RNA SCD-circRNA 2 production. EBioMedicine 45: 155-167, 2019.
36. Chen P, Yue X, Xiong H, Lu X and Ji Z: RBM3 upregulates ARPC2 by binding the 3 'UTR and contributes to breast cancer progression. Int J Oncol 54: 1387-1397, 2019.

37. Fuentes-Fayos AC, Vazquez-Borrego MC, Jimenez-Vacas JM, Bejarano L, Pedraza-Arévalo S, L-López F, Blanco-Acevedo C, Sánchez-Sánchez R, Reyes O, Ventura S, et al: Splicing machinery dysregulation drives glioblastoma development/aggressiveness: Oncogenic role of SRSF3. Brain 143: 3273-3293, 2020.

38. Melling N, Bachmann K, Hofmann B, El Gammal AT, Reeh M, Mann O, Moebius C, Blessmann M, Izbicki JR and Grupp K: Prevalence and clinical significance of RBM3 immunostaining in non-small cell lung cancers. J Cancer Res Clin Oncol 145: 873-879, 2019.

39. Boman K, Segersten U, Ahlgren G, Eberhard J, Uhlén M, Jirström K and Malmström PU: Decreased expression of RNA-binding motif protein 3 correlates with tumour progression and poor prognosis in urothelial bladder cancer. BMC Urol 13: 17, 2013.

This work is licensed under a Creative Commons

Attribution-NonCommercial-NoDerivatives 4.0 International (CC BY-NC-ND 4.0) License. 